EPJ Web of Conferences 58, 01014 (2013)

DOI: $10.1051 /$ epjconf/20135801014

(C) Owned by the authors, published by EDP Sciences, 2013

\title{
Entanglement and Quantum non-locality: an experimental perspective
}

\author{
Alessio Avella ${ }^{1,2}$, Marco Gramegna ${ }^{1}$, and Marco Genovese ${ }^{1, a}$ \\ ${ }^{1}$ INRIM - Istituto Nazionale di Ricerca Metrologica, Strada delle Cacce 91, 10135 Torino (Italy) \\ ${ }^{2}$ Università degli Studi di Torino, Dipartimento di Fisica, Via Pietro Giuria 1, 10125 Torino (Italy)
}

\begin{abstract}
.
The theory of Quantum Mechanics is one of the mainstay of modern physics, a wellestablished mathematical clockwork whose strength and accuracy in predictions are currently experienced in worldwide research laboratories. As a matter of fact, Quantum Mechanics laid the groundwork of a rich variety of studies ranging from solid state physics to cosmology, from bio-physics to particle physics. The up-to-date ability of manipulating single quantum states is paving the way for emergent quantum technologies as quantum information and computation, quantum communication, quantum metrology and quantum imaging. In spite of the impressive matemathical capacity, a long-standing debate is even revolving around the foundational axioms of this theory, the main bones of content being the non-local effects of entangled states, the wave function collapse and the concept of measurement in Quantum Mechanics, the macro-objectivation problem (the transition from a microscopic probabilistic world to a macroscopic deterministic world described by classical mechanics). Problems that, beyond their fundamental interest in basic science, now also concern the impact of these developing technologies. Without claiming to be complete, this article provides in outline the living matter concerning some of these problems, the implications of which extend deeply on the connection between entanglement and space-time structure.
\end{abstract}

\section{Introduction}

Quantum nonlocality has emerged over the last years as one of the most debated and studied aspects of quantum mechanics $(\mathrm{QM})$, since its interest ranges from the very foundations of QM [1,2] to the development of novel quantum technologies, and standing an argument of key relevance for the discussion on the space-time structure [3-11]. To ascribe an inception, the seminal Bell's paper [12] triggered the debate on quantum non-locality demonstrating that, dealing with entangled states, not all the results of quantum mechanics can be mimicked by Local Hidden Variable Theories (LHVT). The extremal bounds fixed by Bell inequalities embody a real dividing line concerning with all those experimental attempts that aim to discriminate between these two facing theoretical frameworks [1], recently intensified by the application to the emerging field of quantum information [13]. These experiments substantially confirmed the standard version of QM. However, while a clear space-like

\footnotetext{
ae-mail: m.genovese@inrim.it
} 
separation has been unequivocally achieved $[14,15]$ and more recently also detection loophole [16, 17] has been eliminated [18-20], a conclusive test is still missing [1] since the two conditions were not realised at the same time, even if not significant chances remain for LHVT.

These achievements fostered the interest for quantum non-locality and, more in general, for quantum correlations.

In the following we present some recent progresses in studying quantum non-locality and related hypothesis of Bell's theorem.

\section{Locality}

The exact meaning and nature of non-locality, enacting non-local influence between space-like separated systems, is matter of a large ongoing controversy [1, 21-27].

Locality as conceived in Bell's theorem means that the joint probability $p(a, b \mid A, B, x)$ of obtaining results $a, b$ when two independent observers, usually dubbed Alice and Bob, perform a measurement A, B respectively can be factorized in

$$
p(a, b \mid A, B, x)=p(a \mid A, x) p(b \mid B, x)
$$

in which $x$ enacts the role of the "hidden variable", distributed with probability density function $r(x)$, and that determines the outcome either deterministically or probabilistically. A violation in Bell inequalities is due to the non-local features of Einstein-Podolsky-Rosen (EPR) entangled particles. On the consequence of this, non-locality violates the separability condition reported in rule (1), even if superluminal transmission (or signalling) has to be excluded, as rigorously demonstrated in [28]. Somehow a clear definition of what quantum non-locality is was given by Stapp: "non-locality property of quantum mechanics is the logical need for information about a choice of experiment freely made in one region to be present in a second region that is space-like separated from the first" [30]. The $x$ variable shared by two measured physical systems because of their generation from a certain common source (as in the case of an EPR pair of photons from the non-linear crystal where the biphoton itself was generated) is conceived like a "property" whose weird influence reveals itself in the case the two measurements take place as space-like separated events, giving back outcomes that depend locally on the measurement settings and on $x$ itself.

In Ref. [25] it is demonstrated that when the above situation occurs, if two independent sources produce two different couples of entangled states, respectively with two independent sets of hidden varialbles $x_{1}, x_{2}$, it sufficies a visibility higher than $50 \%$ in entanglement swapping [31] to falsify these models (to be compared with $71 \%$ of Bell inequalities tests). The variable $x$ in general can be interpreted as the physical state of the two systems [21], or directly of the whole universe.

On the consequence the measurements $A, B$ in Eq. (1) do not depend on the variable $x$ only, or more in the details $x$ can be a non-local beable with the only condition that the outcome probabilities (or "propensities" as artfully clarified in [21]) are determined by the local inputs $A, B$ and the global state $x$. Furthermore Ref. [21] points out that whether one considers a deterministic model (or with epistemic probabilities), one gets in trouble with independence of outcomes from reference frame.

One first question is whether quantum non-locality can be eventually explained by a faster than light hidden influence. Some years ago it was experimentally demonstrated indeed this would require a transmission of information more than ten thousand times the speed of light in the vacuum [32]. Then, very recently, it has been shown theoretically that such a hidden influence, when propagating to a finite velocity in a preferred reference frame, always allows faster than light communication [34] (this is demonstrated for 4-partite case, while it would not be true for bipartite ones). Incidentally, 
one must notice that this results do not affect models where one can suppose that correlations do not propagate continuously in space, as de Broglie-Bohm or GRW models [1].

Finally, Ref. [33] has demonstrated, considering a class of symmetric 3 qubit states, that Bell non-locality does not imply distillability.

Other types of non-locality can be considered beyond Bell non-locality. In particular it is interesting to investigate the connection between Bell non-locality and entanglement. Ref. [35] marks the difference between entanglement and Bell non-locality, showing that entangled states that do not violate any Bell inequality exist, while in Ref. [36] it was shown that maximally non-local quantum states are not the maximally entangled ones, in the sense that: i) in presence of detection inefficiency under a certain threshold ( $82 \%$ ) only non-maximally entangled state still violate Bell inequalities (up to $66 \%$ ) [16]; ii) the Kullback-Leibler distance with the closest local distribution [37] is larger for non-maximally entangeld states; iii) when trying to simulate entanglement with non-local resources the situation becomes more difficult for non-maximally entangled states than for maximally ones. Recently, a strict tie between entanglement and non-locality was anyway demonstrated, by showing that all entangled states, namely, all non-fully-separable states of arbitrary Hilbert space dimension and arbitrary number of parties, violate a Bell inequality when combined with another state which on its own cannot violate the same Bell inequality [38].

An intermediate class of non-locality [27] between the former two, is steering that will be discussed later. It is worth noticing that these three classes of non-locality are equivalent for pure bipartite states.

More recently it was shown that for any entangled state $\rho_{A B}$, there exists another state $\sigma_{A B}$ not violating Bell inequalities, but such that the combination of both states is nonlocal [39, 40]. This result suggests that entangled states always have some hidden nonlocality that can be activated. It remains unclear how to interpret this extra resource in the context of possible applications such as communication complexity or cryptography. In Ref. [41] it was shown that interconversion of quantum states by means of local operations and shared randomness yield in a suitable class of nonlocal games for which it is possible to quantify the non-local content of every entangled state. In this paper, the introduced semiquantum generalization of the usual Bell scenario makes much tighter the relation between nonlocality and entanglement [41]. Instead of providing the measurement devices with classical instructions in fact, it is considered a situation in which these instructions are written on quantum systems. If distinct instructions correspond to distinguishable quantum states, the situation is not different than before. But things become more interesting when the measurement instructions correspond to nonorthogonal states that cannot be perfectly distinguished.

Another interesting approach appeared recently in [42] where it was shown that only those composite quantum systems possessing nonvanishing quantum correlations (quantified by geometric measure of discord [43]) have the property that any nontrivial local unitary evolution changes their global state; a type of nonlocality occurring also for states that do not violate a Bell inequality, such as, for instance, Werner states with a low degree of entanglement.

Non-locality can be quantified $[44,45]$ by determining the minimum number of bits to be communicated between Alice and Bob for reproducing quantum correlations. In Ref. [46] it was shown that one single bit suffices for reproducing the correlations obtained by projective measurements on a two qubits maximally entangled state. However, it remains open the question whether one bit is enough even for partial entanglement. Various alternative approaches can even be covered for quantifying non-locality. In this sense, the method of Colbeck and Renner explits the possibility of giving biased outputs as seen from an adversary point of view [47]. They in fact define local and non-local HV such that when ignoring the non-local hidden variables the experimental outcomes depends on local parameters only, i.e. local hidden variables and settings of measurement apparatuses. With this 
definition they demonstrate that any HVT with a local component cannot reproduce all the results of QM.

The proposal by Elitzur, Popescu and Rohrlich [48] is to quantify non-locality, given a quantum state $r$, by expressing the quantum probability $p(a, b \mid A, B, r)$ in terms of a local part $p_{L}(a, b \mid A, B, r)$ (i.e. satisfying Bell inequalities) and a non-local one $p_{N L}(a, b \mid A, B, r)$, independent of measurements setting and outcomes:

$$
p(a, b \mid A, B, x)=P_{L}(r) \cdot p_{L}(a, b \mid A, B, r)+P_{N} L(r) \cdot p_{N L}(a, b \mid A, B, r),
$$

and determining the decomposition that minimizes $P_{L}$. It turns out that fully non-local correlations $P_{L}=0$ are obtained for bipartite maximally entangled states. Ref. [49, 50] report the cases for non-maximally pure bipartite and tripartite states. In [51] it was considered the $P_{N L}$ part, providing a simulation of this nonlocal correlation using only nonlocal, but no-signaling resources and demonstrating, therefore, that the correlations of all pure entangled states of two qubits can be simulated without communication, hence using only no-signaling resources. Incidentally, in Ref. [52] a similar decomposition has been applied to contextuality.

In Ref.[53] it has been demonstrated that for bipartite non maximally entangled states

$$
\cos (\theta / 2)|00\rangle+\sin (\theta / 2)|11\rangle
$$

the maximal $P_{L}=\cos (\theta)$.

An extension of this scheme has been described in Ref. [54] where a study on non-locality distillation is made, defining distillable NL, i.e. the amount of NL that can be extracted by $\mathrm{N}$ copies of a quantum system, and NL cost, i.e. how much NL is needed for buildining $\mathrm{N}$ copies of the quantum state. For certain undistillable correlations it is shown that the NL cost is strictly larger than distillable NL, an effect termed bound NL in analogy to bound entanglement. Experimental bounds on the local contents of quantum correlations obtained in several Bell inequalities experiments have been discussed in Ref. [55].

An interesting change of perspective with respect to an orthodox approach to QM has been explored in Ref. [56], where Popescu and Rohrlich flip the role of indeterminism and nonlocality, considering a box (PR box) that relates Alice's and Bob's outputs with their inputs A, B with the only request of compatibility with special relativity, i.e. preserving relativistic causality:

$$
\sum_{b} p(a, b \mid A, B)=p(a \mid A)
$$

and analogously summing over $a$. This represent a convex set of probabilities (being combinations of no-signalling probabilities still non-signalling) with a final number of extremal points, i.e. a polytope. In Ref. [57] it was shown as this polytope has only 8 vertices more than the local polytope (i.e the one satisfying Eq. 4).

It is possible to consider the following case where the relation between outputs and inputs is defined as

$$
a \bigoplus b=A \cdot B
$$

as a specific example of PR box, where $\bigoplus$ stands for sum modulo 2. Furthermore, Alice's and Bob's marginal distributions are completely random and relativistic causality is satisfied (i.e. Alice and Bob cannot communicate directly by exploiting PR boxes.) It can be shown that PR boxes allow for stronger non-local correlations than QM, leading for instance to a violation of CHSH inequality of $S=4$ to be compared with the Tsirelson bound [58] $S=2 \sqrt{2}$ of QM and $S=2$ of a classical theory. 
Therefore, QM correlations do not produce a violation of locality as strong as allowed by causality (it is worth noticing this is true for two dimensional systems, Ref. [59] shows how the situation changes in the case of higher dimensionality). On the consequence it is important to fathom the possibility of a physical principle reducing these "superquantum" correlations [56] to QM correlations.

A first contribution that helped to shed light on this analisys has been reported in Ref. [60], in which the balance between correlations and communication complexity [57] has been explored. In particular, when Alice and Bob want to compute some Boolean function $F(A ; B)$ of input $A$, known to Alice only, and input $B$, known to Bob only, their concern is minimizing communication between them needed from Alice for knowing the answer. This problem is told to be trivial when one bit communication suffices. In Ref. [60] it was shown that the requirement of nontrivial communication complexity bounds to $90.8 \%$ the success probability of PR boxes operating in a probabilistic way, a limit that, anyway, is still larger than the one imposed by Tsirelson bound, $85.4 \%$ (75\% being the classical limit). Therefore, the non-triviality of communication complexity (i.e. the existence of both hard and easy communication complexity problems) can be interpreted as a partial characterization of non-local correlations achievable by measurements on entangled systems. In this context it is worth mentioning that, even if QM is limited by Tsirelson bound, it has been experimentally demonstrated in Ref. [65] that acting a postselection operation the data can approach the PR limit [61-64].

On the other side, various properties common to all the theories not allowing for superluminal signalling and predicting the violation of Bell inequalities are taken into account in Ref. [66], between them: intrinsic randomness, uncertainty due to incompatibility of two observables, monogamy of correlations ${ }^{1}$, impossibility of perfect cloning, privacy of correlations (if two honest parties know to share correlations with some degree of monogamy, they can estimate and possibly bound their correlations with a third dishonest party, the eavesdropper), bounds in the shareability of some states ${ }^{2}$. In this regard, it was shown that for any distribution, the properties of nonlocal, no arbitrarily shareable, and positive secrecy content are equivalent.

A further relevant argument has been raised up in Ref. [67], where it has been deduced how QM cannot be more non-local with measurements that respect the uncertainty principle, demonstrating the link between these two central properties of QM. In detail, it was examined the game where Alice and Bob win when their answer satisfies relation 5 between outputs and inputs. Then the reasoning focused on the concept of steerability (i.e. what states Alice can prepare on Bob's system remotely). If $\rho_{A B}$ represents a state shared by these two parties, the reduced density matrix pertaining Bob's system results to be:

$$
\rho_{B}=\Sigma_{a} p(a \mid s) \rho_{s, a}
$$

and as Schrödinger noticed, for each experimental setting $s$ there exists a measurement on Alice's system that allows to have the state $\rho_{s, a}$ with probability $p(a \mid s)$ on Bob's site, where $a$ indicates the measurement outcomes. When an average operation is performed for each of Alice's settings and over all Alice's measurement outcomes, Bob's state remains exactly the same, this resulting in the evidence that a steering procedure does not allow to any no-signalling violation. So, expressing with $\zeta_{s, a}$ the bound given by the maximization over a proper set of "fine-grained" uncertainty relations (directly

\footnotetext{
${ }^{1}$ (i.e. at variance with classical ones, that cannot be shared among an indefinite number of parties: when an entangled state is shared between three parties, e.g. Alice, Bob and Clare, and at the same time Alice and Bob maximally violate a Bell inequality, than Alice and Clare are completely uncorrelated, this derives by extremality conditions on a polytope

${ }^{2}$ In the analysis of the monogamy of correlations, the shareability represents a natural property. In fact, a bipartite probability distribution $P(a, b \mid A, B)$ is said to be $\mathrm{m}$ shareable with respect to Bob, if there exists an (m+1)-partite distribution $P\left(a, b_{1}, \ldots, b_{m} \mid A, B_{1}, \ldots, B_{m}\right)$ being symmetric with respect to $\left(b_{1}, B_{1}\right) \ldots\left(b_{m}, B_{m}\right)$, with marginals $P\left(a, b_{i} \mid A, B_{i}\right)$ equal to the original distribution $P(a, b \mid A, B)$.
} 
related to the ones expressed in entropic form [67]), a tight bound for the game success is inferred:

$$
P_{\max }^{\text {game }}=\Sigma_{s} p(s) \Sigma_{a} p(a \mid s) \zeta_{s, a}
$$

where it must be taken into account that whenever $\zeta_{s, a}<1$, it is not possible to obtain a measurement outcome with certainty for all the measurements simultaneously.

Experimental evidencies of steering correlations have been achieved by measuring violation of inequalities [68, 69] (even eliminating detection loophole [70] by exploiting highly efficient TES (transition-edge sensors) Photon Number Resolving detectors) casted in terms of inferred variances of complementary observables [71, 72]. Demanding completeness, some entangled state does not violate the inequalities suggested in [71]: a criterium based on Shannon entropy, violated by more states, has been proposed in [73].

Following this idea, in [74] was posed the question if complementarity is tied in general to nonlocality. First of all it was pointed out how one can attribute different operational meanings to the concept of joint measurability of two observables, $\mathrm{A}$ and $\mathrm{B}$ : i) there is an observable $\mathrm{C}$ such that its measurement allows deducing $\mathrm{A}$ and $\mathrm{B}$ values (the probability distribution of $\mathrm{C}$ outcomes contains those of $\mathrm{A}, \mathrm{B}$ as marginals); ii) in the measurement sequence $\mathrm{ABA}$, the two $\mathrm{A}$ measurements give the same result; iii) the measurement sequence $A B$ produces the same probability distribution over $B$ outcomes as a direct B measurement. For QM these three statements are equivalent, but in Ref. [74] it is demonstrated that while any no-signalling theory that does not display complementarity in the sense of property iii) admits a local realistic description, this is not true for properties i) and ii) (this is shown by two toy models reproducing PR box correlations).

It is also important to account for the reasoning about whether the PR box can be considered as a "unit of non-locality". In regard of this Ref. [75] illustrated that PR boxes cannot simulate correlations from cluster states and, thus, on the one hand, they cannot be considered as a "unit of non-locality" for multipartite states and, on the other side, this result demonstrates that the structure of non-local correlations is rather different from the structure of entanglement.

Various further studies on the properties of PR boxes can be found in [76-79]. In this context, recently it has been shown that the request of covariance for an extension of QM is equivalent to a local HVT for deterministic models [80].

Finally, a further question is whether the nonlocality of a global (combined) state of several entangled states can be simply derived from the nonlocalities of each individual state, i.e. if non-locality is additive. The answer to this question has been given by Ref. [81]. In this paper, by considering unbounded Bell inequalities (i.e. Bell inequalities such that by proper choice of states and measurements, this quantum versus classical separation can become arbitrarily large [82]), it was shown that by combining (i.e. making their direct product) many local entangeld states one can obtain a non-local one (superactivation of non-locality), i.e. non-locality is not additive. This result rises the question whether superactivation of non-locality is possible for any entangled state that admits a local representation. A first step in this sense has been the recent demonstration that all entangled states that are useful for teleportation can be superactivated [83] establishing a firm link between teleportation and nonlocality.

\section{Realism and determinism}

An other relevant concept often quoted as an hypothesis at the basis of the demonstration of Bell inequalities is Realism, that following the reasoning of Ref. [21] means that measurement results reflect preexisting properties. This should not be confused with determinism, i.e. that measurements 
results are fixed a priori by some hidden variables (HV). Indeed a realistic model can be casual, i.e. the preexisting properties do not determine the outcome, but its probability.

An example of a realistic, but not deterministic model, has been proposed by Leggett in [22] where pre-existing properties should be pure quantum states and that these pure states define the local probabilities, model that has been experimentally falsified [23].

In Ref.[86, 87] by studying an entanglement-assisted delayed-choice experiment it was demonstrated that, in the absence of superluminal communication, realism (defined as a property of photons being either particles or waves, but not both) is incompatible with determinism, even if the hidden variable theory satisfying one of these properties reproduces quantum predictions.

Another interesting result concerning determinism is that measurements outcomes leading to a violation of Bell inequalities cannot be perfectly predicted within a non-signalling theory: any theory satisfying the non-signalling principle and being non-local (in Bell sense) is also necessarily intrinsically random $[66,95]$. This relation introduces the question about the existence of a direct relation between the amount of Bell inequalities violation and the randomness produced in the experiment. In Ref. [84] it is shown how the amount of randomness certified by non-local quantum correlations is non-equivalent both to entanglement and to non-locality (intended as violation of Bell inequalities) by showing that quantum correlations with arbitrary little non-locality and states with arbitrary little entanglement can be used to certify that close to the maximum two bits of randomness are produced (the maximal violation of $\mathrm{CHSH}$ inequality guaranteeing only 1.23 bits of randomness), even if hierarchically entanglement is needed for non-locality, and non-locality is necessary for certifying randomness.

Always in this context, in Ref. [85] it was shown the existence of N-party Bell tests attaining maximal global randomness, that is, where a combination of measurements by each party provides $\mathrm{N}$ perfect random bits.

\section{Random choice}

Bell's theorem enclose in itself Free Will as an implicit assumption. On the contrary a deterministic theory describing an observer rules out any chance of free will. In fact, one can envisage the three alternatives of observers endowed with free will, solipsism and of a completely deterministic world, where pre-determinism drives the choices of the observers. It can be taken into account also a deterministic theory, where free will requires an extra-physical observer or evenly a non deterministic theory in which there is no free will. It was suggested that free will can merge in a probabilistic theory, as quantum mechanics with wave function collapse (see the discussion in Ref. [88]). However, in our opinion, this would lead at most to a random choice, not to what is usually considered both in phylosophy or in common language "free will" (i.e. the possibility of the observer to "decide" autonomously and in complete freedom which action to accomplish).

Nonetheless, restricting the analysis to Bell's theorem, one has to specify clearly what free will is in this context. Here free will means that the measurement settings can be chosen at random (i.e. they are not determined a priori). Indeed, it is not needed a choice of an observer, a real random number generator without any predetermination of outcomes suffices (that indeed is what happens in a real experiments). In the consequence of this, at odds with the term "free will" that can be misleading (and can also induce some confusion outside physicists community [89], leading to the need to carefully choose the way how certain statements are expressed), "random choice" could be a more relevant choice. Of course an intrinsically "true" random number generator suffices, standing that one must be sure that the generated probabilities cannot be determined by some "hidden variable". An experiment whose outcomes violate Bell inequolities satisfies this requirement. 
A quantification of this kind of "free will" has been taken into account in Ref. [90] by means of a scheme like the following: given $\mathrm{N}$ possibilities over which a choice has to be made, an amount of $\log _{2}(N)$ bits of free will is needed. Moreover, in the case the choice is constrained to $M<N$ possibilities, the consequent lack of free will is $\log _{2}(N)-\log _{2}(M)$. By exploiting the connection with non-locality quantification (refer to [46]), it has been demonstrated that for maximally entangled bipartite states all correlations by the lack of a single bit of free will at one side (e.g. Alice), while for partially entangled bipartite systems the lack of two bits of free will on the other, render Bell inequality violation impossible (independently from alphabet size), but it is yet not known what happens with the lack of one single bit. Ref. [90] pointed out also that the connection between lack of free will and detection loophole must still be explored.

A different way for quantifying the random choice was provided by [92, 93] (see also [94]). Reconsidering Eq. 4, one can write:

$$
p(a, b \mid A, B)=\int d x p(a, b \mid A, B, x) r(x \mid A, B)
$$

when $r(x \mid A, B)=r(x)$ one has perfect random choice (no lack of "free will" in the Ref. [90, 92, 93] terminology). Then the degree of measuremet dependence may be quantified by the variational distance between the distribution of the shared parameter for any pair of measurement settings [92]:

$$
M=\sup _{A ; A^{\prime} ; B ; B^{\prime}} \int d x\left|r(x \mid A, B)-r\left(x \mid A^{\prime}, B^{\prime}\right)\right|
$$

In Ref. [91] it is shown as 59\% measurement independence is optimal for simulating singlet correlation, when the hidden variables distribution depends on measurements on one party only, while $86 \%$ is optimal [92] when any model is considered.

\section{References}

[1] M. Genovese, Phys. Rep. 413, 319-398 (2005).

[2] M. Genovese, Adv. Sci. Lett. 3, 249-258 (2010).

[3] D. Ahn et al., arXiv:1207.6062 (2012).

[4] Y. Aharonov et al., arXiv:1206.6224v5 (2012).

[5] S. Lloyd et al., Phys. Rev. Lett. 106, 040403 (2001).

[6] O. Oreshkov et al., Nat. Comm. 10, 1038 (2012).

[7] W. K. Wootters, Int. Jour. Th. Phys 23, 701 (1984).

[8] M. Genovese, Advanced Science Letters 2, 303 (2009).

[9] H. Nikolic, Advanced Science Letters 17, 271 (2012).

[10] M. D'Ariano, Advanced Science Letters 17, 130 (2012).

[11] E. Anderson, Ann. Phys. 524, 757 (2012).

[12] J. S. Bell, Physics 1195 (1965).

[13] F. Bennati et al., Quantum Information, Computation and Cryptography (Springer, Berlin, 2010).

[14] W. Tittel et al.,Phys. Rev. A 59, 4150 (1999).

[15] G. Weihs et al., Phys. Rev. Lett. 81, 5039 (1998).

[16] P. H. Eberhard, Phys. Rev. A 47, R747, 2800-2811 (1993).

[17] G. Brida et al., Phys. Lett. A 268, 12-16 (2000). 
[18] M. A. Rowe et al., Nature 409, 791 (2001).

[19] M. Giustina et al., Nature 497, 227 (2013).

[20] B. G. Christensen et al., arXiv:1306.5772v1 (2013).

[21] N. Gisin, arXiv:1012.2536v1 (2010).

[22] A. J. Leggett, Found. of Phys. 33, 1469 (2003).

[23] S. Gröblacher et al., Naure 446, 871 (2007).

[24] C. Branciard et al., Nature Phys. 4, 681 (2008).

[25] M. Zukowsky et al., Phys. Rev. Lett. 71, 4287 (1993).

[26] A. Khrennikov, International Journal of Quantum Information 7, 71 (2009).

[27] H. M. Wiseman et al., Phys. Rev. Lett. 98, 140402 (2009).

[28] G. C. Ghirardi, A. Rimini and T. Weber, Lett. Nuov. Cim. 27, 293 (1980).

[29] J. Barrett et al., Phys. Rev. A 71, 022101 (2005).

[30] H. P. Stapp, Found. of Phys. 42, 647 (2012).

[31] C. Branciard et al., Phys. Rev. Lett. 104, 170401 (2010).

[32] D. Salart et al., Nature 454, 861 (2008).

[33] T. Vertesi and N. Brunner, Phys. Rev. Lett. 108, 030403 (2012).

[34] J. Bancal et al., Nat. Phys. 8, 867 (2012).

[35] R. F. Werner, Phys. Rev. A 80, 4277 (1989).

[36] A. A. Methot and V. Scarani, Quant. Inf. Comput 7, 157 (2007).

[37] A. Acin et al, Phys. Rev. Lett. 95, 210402 (2005).

[38] Y. Liang et al., arXiv:1210.0548v2 (2012).

[39] L. Masanes, Y. C. Liang and A. C. Doherty, Phys. Rev. Lett. 100, 090403 (2008).

[40] M. Navascues and T. Vertesi, Phys. Rev. Lett. 106, 060403 (2011).

[41] F. Buscemi, Phys. Rev. Lett. 108, 200401 (2012).

[42] S. M. Giampaolo et al., arXiv:1206.4075v3 (2013).

[43] B. Dakizc, V. Vedral, and C. Brukner, Phys. Rev. Lett. 105, 190502 (2010).

[44] G. Brassard et al., Phys. Rev. Lett. 83, 1874 (1999).

[45] M. Steiner, Phys. Lett. A 270, 239 (2000).

[46] B. F. Toner and D. Bacon, Phys. Rev. Lett. 91, 187904 (2003).

[47] R. Colbech and R.Renner, Phys. Rev. Lett. 91, 050403 (2008).

[48] A. C. Elitzur, S. Popescu and D.Rohrlich, Phys. Lett. A 162, 25 (1992).

[49] V. Scarani, Phys. Rev. A 77, 042112 (2008).

[50] C. Branciard, N. Gisin and V. Scarani, Phys. Rev. A 81, 022103 (2010).

[51] N. Brunner, Phys. Rev. A 78, 052111 (2008).

[52] E. Anselem et al., arXiv:1111.3743v3 (2012).

[53] S. Portmann, C. Branciard and N. Gisin, Phys. Rev. A 86, 012104 (2012).

[54] N. Brunner et al., Phys. Rev. Lett. 106, 020402 (2011).

[55] L. Aolita et al., Phys. Rev. A 85, 032107 (2012).

[56] S. Popescu and D. Rhorlich, Found. Phys. 24, 379 (1994).

[57] H. Buhrman et al., Rev. Mod. Phys. 82, 665 (2010).

[58] B. S. Tsirelson, Lett. Math. phys. 4, 93 (1980).

[59] A. Cabello, Phys. Lett. A 337, 64 (2012).

[60] G. Brassard et al., Phys. Rev. Lett. 96 ,250401 (2006).

[61] N. Gisin, Phys. Lett. A 210, 151 (1996). 
[62] S. Marcivitch et al., Phys. Rev. A 75, 022102 (2007).

[63] Y. Chen et al., Phys. Rev. Lett. 97, 170408 (2006).

[64] A. Cabello, Phys. Rev. Lett. 88, 060403 (2002).

[65] D. S. Tasca et al., Phys. Rev. A 80, 030101 (2009).

[66] L. Masanes, Phys. Rev. A 73, 012112.

[67] J. Oppenheim and S. Wehner, Science 330, 1072 (2010).

[68] Z. Y. Ou et al., Phys. Rev. Lett. 68, 3663 (1992);

[69] J. C. Howell et al., Phys. Rev. Lett. 92, 210403 (2004).

[70] D. H. Smith et al., Nat. Comm. 3, 625 (2012).

[71] M. D. Reid, Phys. Rev. A 40, 913 (1989).

[72] M. D. Reid et al., Rev. Mod. Phys. 81,1727 (2009).

[73] S. P. Walborn et al., Phys. Rev. Lett. 106, 130402 (2011).

[74] T. Fritz, Phys. Rev. A 85, 022102 (2012).

[75] J. Barrett and S. Pironio, Phys. Rev. Lett. 95, 140401 (2005).

[76] A. Short et al., Phys. Rev. A 73, 012101 (2006).

[77] J. Barrett, Phys. Rev. A 75, 032304 (2007).

[78] I. Pitowsky, Phys. Rev. A 77, 062109 (2008).

[79] N. Linden et al., Phys. Rev. Lett. 99, 180502 (2007).

[80] N. Gisin, Phys. Rev. A 83, 020102 (2011).

[81] C. Palazuelos, Phys. Rev. Lett. 109, 190401 (2012).

[82] M. Junge, C. Palazuelos, D. Perez-Garcia, I. Villanueva, and M. M. Wolf, Phys. Rev. Lett. 104, 170405 (2010).

[83] D. Cavalcanti, A. Acin, N. Brunner, and T. Vertesi, arXiv:1207.5485 (2012).

[84] A. Acin et al., Phys. Rev. Lett. 108, 100402 (2012).

[85] C. Dhara, G. Prettico and A. Acin, arXiv:1211.0650 (2012).

[86] R. Ionicioiu, T. Jennewein, R. B. Mann, D. R. Terno, arXiv:1211.0979v1 (2012).

[87] R. Ionicioiu, T. Jennewein, R. B. Mann, D. R. Terno, arXiv: 1301.6969v1 (2013).

[88] G. Brassard and P. Raymond-Robichaud, arXiv:1204.2128v1 (2012).

[89] P. Cary, Behav. Scienc. \& the Law 25, 165 (2007).

[90] J. Barrett and N.Gisin, Phys. Rev. Lett. 106, 100406 (2011).

[91] M. Banik et al., arXiv:1204.3835v1 (2012).

[92] M. J. W. Hall, Phys. Rev. Lett. 105, 250404 (2010).

[93] M. J. W. Hall, Phys. Rev. A 82, 062117 (2010).

[94] G. Kar, Journ. Phys. A 44, 152002 (2011).

[95] A. Valentini, Phys. Lett. A 297, 273 (2002). 